\title{
RESEARCH
}

Open Access

\section{Hybrid medical simulation - a systematic literature review}

\author{
Wayne J. Brown ${ }^{*^{*}}$ (I) and Richard A. W. Tortorella ${ }^{2}$
}

\author{
*Correspondence: wayneb@ \\ student.uef.fi \\ ${ }^{1}$ University of Eastern Finland, \\ Yliopistokatu 2, Joensuu FI-80100, \\ Finland \\ Full list of author information is \\ available at the end of the article
}

\begin{abstract}
Health-care education based upon technology enabled mannequins (high-fidelity simulators) is a costly investment for colleges and universities. However, a hybrid model using wearable technology integrated with human actors (standardized patients) may present a cost-effective alternative to high fidelity simulation training scenarios. A systematic literature review of papers published from 1960 to 2019 illustrates that hybrid simulation can be as effective as high fidelity simulators in certain training scenarios while at the same time providing a superior training context to enhance learners patient to care-giver interactions and to better immerse the trainee in the feelings and emotion of the scenario.

Keywords: Standardized patient, Human actor, High-fidelity patient simulator, Wearable technology, Hybrid simulation
\end{abstract}

\section{Introduction}

Many health care training institutions lack the financial means to purchase high fidelity patient simulators. As a result, faculty and staff are often left to improvise a simulation based training solution using existing equipment combined with supplemental, sometimes non-standard, materials. This approach may put students graduating from these institutions at a disadvantage to those students who attend more affluent institutions with modern simulation equipment. A hybrid simulation approach may provide colleges and universities with limited budgets with a more affordable simulation option, while at the same time providing a more effective training experience. Indeed, Lous et al. found through a systematic literature review that considering technical aspects of obstetrical emergencies management, hybrid simulation training is as efficient as highfidelity training (Lous et al., 2020).

The purpose of this literature review is to survey existing research in the use of hybrid simulation in health care education to determine the current role this form of simulation plays and in particular, the advantages and disadvantages of using hybrid simulation as compared to high fidelity simulation or standardized patients only.

The use of simulators in health care education covers a wide spectrum of medical education disciplines, including but not limited to anesthesia, emergency medicine, and surgery (Schubart et al., 2012). Indeed, for nursing and midwifery education,

(c) The Author(s). 2020 Open Access This article is licensed under a Creative Commons Attribution 4.0 International License, which permits use, sharing, adaptation, distribution and reproduction in any medium or format, as long as you give appropriate credit to the original author(s) and the source, provide a link to the Creative Commons licence, and indicate if changes were made. The images or other third party material in this article are included in the article's Creative Commons licence, unless indicated otherwise in a credit line to the material. If material is not included in the article's Creative Commons licence and your intended use is not permitted by statutory regulation or exceeds the permitted use, you will need to obtain permission directly from the copyright holder. To view a copy of this licence, visit http://creativecommons.org/licenses/by/4.0/. 
simulation has become indispensable as an alternative to hands-on experience with real-life patients ("Andersen, Downer, O’Brien, \& Cox, 2019).

High-fidelity simulators are life-size mannequins that can simulate multiple human functions such as breathing, generating a pulse, producing a heartbeat as well as being able to communicate with the learner through a remote operator interface (Goolsby, Goodwin, \& Vest, 2014). Low-fidelity simulators on the other hand, which are sometimes referred to as partial or table-top simulators, are typically designed to simulate a specific aspect of the human anatomy such as an arm to practice IV starts (Goolsby et al., 2014). Simulators provide a safe, relatively risk free context for learning and has been for many years an alternative for learning on actual patients (Sanko, Shekhter, Rosen, Arheart, \& Birnbach, 2012).

Standardized patients, or human actors, are on the opposite end of the simulation spectrum. Standardized patients are typically professional actors or readily available students or volunteers trained to simulate a variety of medical problems in a 'consistent, reliable, realistic and reproducible manner' (Verma, Bhatt, Booten, \& Kneebone, 2011). The use of human actors increases the realism of the training, particularly from the perspective of patient-caregiver interactions, and further immerses the learner into the feelings and emotion of the learning experience ("Dunbar-Reid, Sinclair, \& Hudson, 2015; Verma et al., 2011).

It is interesting to note that the term hybrid is not well defined in the literature, and can cover a wide variety of meanings. For example, hybrid could mean the close integration of human actors with technology in the form of a wearable device or the use of a human actor and a high fidelity simulator, side by side, in the same scenario - but as independent learning modalities that represent the same patient and therefore the whole of the training scenario. Indeed, Lous et al. defines hybrid simulation as the use of two or more simulation modalities within the same simulation session (Lous et al, 2020). However, hybrid simulation, for the purposes of this paper, is defined as the utilization of wearable or augmentative technology in conjunction with a human actor in a health-care education context. The technology typically is used to simulate aspects of a particular medical scenario in which the human actor is not able to simulate or would be at risk to simulate.

Hybrid simulators enable the educator to create a learning scenario that can incorporate human interactions, reactions and body language as well as clinical data such as blood pressure, and stomach sounds which may be controlled by the educator. Hybrid simulation allows both procedural and communication skills training, bringing a sense of realism to the training that may not be attained by using human actors or simulators alone. The use of hybrid simulation can be a cost-effective training option compared to high fidelity simulators exclusively, as these simulators can cost upwards to tens of thousands of dollars (Amerjee, Akhtar, Ahmed, \& Irfan, 2018).

As per the Guide to Conducting a Systematic Literature Review of Information Systems Research published by Okoli and Schabram, the following eight steps were used as a roadmap for this research:

1 Purpose of the literature review.

2 Protocol for training.

3 Searching for the literature. 
4. Practical screen.

5 Quality appraisal.

6 Data extraction.

7 Synthesis of studies.

8 Writing the review (Okoli \& Schabram, 2010).

\section{History of simulation in healthcare}

Prior to the 1900's, healthcare education was primarily executed through apprenticeship and mentoring (Rosen, 2008). In the early 1900's, trainees were more formally educated on scientific principles and later on were measured against knowledge, skills and behaviours (Rosen, 2008). Toward the end of the twentieth century, human patient simulation was introduced. This represented a significant milestone in the evolution of health sciences education (Rosen, 2008). Many innovations helped facilitate the advancement of health education simulation technology as we know it today. Advances in flight simulation, technology in general and manmade materials all played a role in the advancement of healthcare education (Rosen, 2008). Simulators were first used in the medical field to train students on the proper use of anesthesia (Wisborg, Brattebo, Brinchmann-Hansen, \& Hansen, 2009). Since that time, simulators have been used extensively in health care education for skills training, decision making as well as individual and team training (Wisborg et al., 2009).

The use of volunteers to act as patients (human actors) began in 1963 by a neurologist from the University of Southern California (Rosen, 2008). This 'novel' approach was used to teach medical students during the third year of their neurology clerkship (Rosen, 2008). However, it took 30 years for the Medical Council of Canada to incorporate a standardized patient examination into licensure in 1993 (Rosen, 2008). Eleven years later the society for simulation in healthcare was established, with the first simulation meeting taking place in January 2006 (Rosen, 2008).

In 2005, human patient simulation was employed in undergraduate medical education at which time medical educators acknowledged that simulation was the future of medical education (Rosen, 2008). Indeed, modern simulation has progressed significantly since its introduction; however, there are still major barriers to its use in health care education (Rosen, 2008).

\section{Defining a standardized patient}

Standardized patients were introduced by Howard Barrows in the 1960's (Yudkowsky, 2002). Since that time extensive research has been conducted in the use of standardized patients for the purposes of testing, measurement and assessment (Yudkowsky, 2002). Rosen, 2008 defines a standardized patient as "actors used to educate and evaluate history taking and physical examination skills, communication, and professionalism." These standardized patients were often used in standardized assessments and were relied upon to educate and evaluate history taking, physical examination skills, communication skills and overall professionalism (Rosen, 2008). Because standardized patients are often used in assessment scenarios it is critical that the standardized patient can simulate a real patient repeatedly and in a consistent and reliable manner (Yudkowsky, 2002). 
The advantages of standardized patients have been widely reported in the literature. Standardized patients have been found to add further realism to a simulation, creating an approximation of the actual psychological responses experienced during a clinical event (Ignacio et al., 2015). Standardized patients are coached to create authentic emotional responses during the simulated scenario, thus producing realistic patient care scenarios similar to those found in the real world (Luctkar-Flude, Wilson-Keates, \& Larocque, 2012).

However, at the end of the day, a standardized patient is not a real patient. Indeed, a standardized patient is an actor who strives to realistically portray a real patient, thus adding emotional stressors which enhance clinical performance (Ignacio et al., 2015), and providing the learner with a significant degree of high-fidelity, the advantages of which far outweigh the loss of authenticity (Yudkowsky, 2002). However, there does not seem to be agreement in the literature as to what exactly constitutes a standardized patient. Dunbar-Reid et al. define a standardized patient as a person who acts as themselves to assist in staff education ("Dunbar-Reid et al., 2015). In this context, the actor patient truthfully answers questions about their own medical and social history ("Dunbar-Reid et al., 2015). Dunbar-Reid et al. further define a simulated patient as different from a standardized patient in that a simulated patient acts as a patient, portraying specific behaviours and symptoms to align with some pre-determined illness ("DunbarReid et al., 2015). However in both of these contexts, the actor patient does not participate in any form of assessment or evaluation as was common in the past. Yudkowsky posits that a standardized patient is available when and where required and is trained to portray specific cases accurately, repeatedly and consistently (Yudkowsky, 2002). Yudkowsky goes on to define a standardized patient as an actor or 'other lay person' who is 'rigorously' trained to present certain physical symptoms and medical history in a highly consistent way (Yudkowsky, 2002). A well-trained standardized patient will respond accurately yet consistently to trainee questions or procedures regardless of the way in which each trainee approaches the scenario (Yudkowsky, 2002).

However, the biggest downfall of a standardized patient, despite the realism in which he can portray a human patient is their inability to be subjected to invasive procedures such as intubation or insertion of an IV (Wisborg et al., 2009).

As a point of clarity, it is worth pointing out the concept of a virtual patient. In this case the patient is neither a mannequin nor an actor, but a data set belonging to a past real patient that can be presented to the learner as a virtual patient. Bloice et al. defines a virtual patient as unformatted electronic patient records which have been retrieved from a hospital information system in their raw form and are often presented to the learner through a virtual patient user interface (Bloice et al., 2013).

\section{Defining a high-fidelity patient simulator}

On the other end of the simulation spectrum is the high fidelity simulator. This form of simulation provides the trainee with the very thing that a standardized patient cannot; a patient in which one can perform invasive procedures. There is much literature that will support the use of high fidelity simulators to improve knowledge, procedural skills and attitudes of students (Tuzer, Dinc, \& Elcin, 2016). Indeed, the literature confirms that students not only benefit educationally from simulations involving high 
fidelity simulators, but they actually accept this form of simulation. (Smithburger, Kane-Gill, Ruby, \& Seybert, 2012). However, there is also much research to suggest that students find high fidelity simulators lacking the ability to authentically simulate live patients which can provide realistic feedback, sometimes resulting in significantly lower satisfaction levels as compared to other learning modalities (Luctkar-Flude et al., 2012). Never-the-less, students still rate high fidelity simulators as 'somewhat realistic' (Luctkar-Flude et al., 2012). Wallace et al. define high fidelity simulators as computerized mannequins (spelled manikin by some researchers) that can exhibit 'realistic' responses to invasive procedures (Wallace, Gillett, Wright, Stetz, \& Arquilla, 2010) vs a low fidelity simulator which is a full body mannequin that does not provide feedback to the student based upon student interventions (Tuzer et al., 2016).

High fidelity simulators have been used in the past for many aspects of health education from specific medical procedures to developing skills to manage critically ill patients (Kennedy et al., 2013). Luctkar-Flude, Wilson-Keates, \& Larocque found that high fidelity simulation contributes to significant improvement in knowledge, confidence and performance in clinical settings (Luctkar-Flude et al., 2012).

\section{Research methodology}

In 2010, researchers at Concordia University, Canada, published a guide to conducting a systematic literature review for information systems research (Okoli \& Schabram, 2010). This published work provides a detailed framework for writing a systematic literature review that has its roots in information technology. As this systematic literature review is rooted in computer science, it was deemed appropriate to use Okoli's work as the basis for this body of work. Because there was only one reviewer, and as per Okoli's recommendation, a training and protocol document to ensure review consistency was not required.

\section{Research question}

This literature review supports research in the area of hybrid simulation in health care education. The overarching research question is: How can health care education be enhanced through the use of wearable technology and human actors?

\section{Databases employed}

To answer this research question, the authors have chosen the following ten well known and reputable databases in which to base this literature review: Scopus, PubMed, Web of Science, IEEE, ACM, Science Direct, Springer Link, EMBASE, Cochrane Library and CINAHL. Each of these databases has unique advantages when it comes to systematic literature reviews. Indeed, Lawrence (2008) found that valuable literature may be lost if any one single database is used for a literature review and that 'different databases are better suited for some topics than others' (Lawrence, 2008).

PubMed, in particular, was chosen as it is a major bibliographic database (O'MaraEves, Thomas, McNaught, Miwa, \& Ananiadou, 2015) and has been found to be one of the most common databases used for systematic literature reviews (Qi et al., 2013). However, the authors are aware that there is no perfect database, indeed Qi, et al. found that the PubMed database had the highest proportion of wrong issue information 
among the three leading library databases: PubMed, EMBASE and Cochrane (Qi et al., 2013).

IEEE, ACM, Science Direct and Springer Link have been cited as being the most reliable electronic databases that are scientifically and technically peer reviewed (Latif, Abbas, \& Assar, 2014). These databases provide access to high quality proceedings of key conferences and journals in computer science and engineering (Latif et al., 2014).

Scopus was included as a database of choice as it is positioned by its makers as the largest existing database of abstracts and citations available, a fact which aligns with the author's anecdotal information and experience (EBSE, 2007). Similarly, Web of Science, EMBASE, Cochrane Library and CINAHL anecdotally are well-respected and utilized research databases; in particular this experience is supported by the makers of Web of Science which claims that this database contains over 20,000 objectively selected quality journals which include papers that have been cited over 1.4 billion times since the 1900s.

\section{Supplementary procedures}

Appropriate papers were initially identified through traditional searches of electronic databases. A reference search was conducted on the final papers used as the basis for this literature review to identify other papers that may have been missed through traditional literature review techniques. These papers were subsequently analyzed to determine their applicability to the study.

\section{Keywords}

To identify the keywords in which to search the databases, an independent, initial search was performed on each of the seven databases based upon the phrases: 'High Fidelity Patient Simulator' and 'Standardized Patient'. These phrases were arrived at based upon the author's prior readings and understanding of the research topic. No filters were set on any of the databases for this initial search phase. A convenience sample of twenty papers from each result set, as sorted by relevance, was manually analyzed to acquire a tally of the keywords in each paper based upon: 'author's keywords' and the abstract of each paper, where one was present. A spreadsheet was constructed to track the occurrence of each keyword for each database. Once all papers were analyzed, an accumulated total of each keyword was formulated to attain an overall count of the number of occurrences of each keyword. Based upon the number of occurrences and the relevance of each keyword to the research topic, the following eleven keywords were selected to perform a more extensive database search: actor patient, actor victim, simulated patient, standardized patient, trained human actor, high-fidelity, high fidelity, manikin, mannequin, simulator, and wearable. These keywords were eventually integrated into an appropriate search query to identify papers relevant to the research question.

\section{Search query}

As the focus of this research is the use of hybrid simulation, a search query was developed that would produce a result set of papers that addressed both simulation and human actors - thus a hybrid simulation. An appropriate search query was formulated 
that would find the intersection of both fields. The 'OR' operators captured the papers from each field of interest, whereas the 'AND' operator functioned to select papers that met both conditions. The search query used was as follows: ("actor patient" OR "actor victim" OR "simulated patient" OR "standardized patient" OR "trained human actor") AND ("high-fidelity" OR "high fidelity" OR "manikin" OR "mannequin" OR "simulator" $O R$ "wearable"). Each database was tested to determine the unique implementation of Boolean operators for that database. Once the authors understood this implementation, the search query was syntactically tuned to produce consistent results.

\section{Inclusion/exclusion criteria}

The abstract of each paper from the initial search result-set was reviewed, and when necessary the entire paper was read, to determine if the paper was to be included in the literature review. Each paper which met the inclusion criteria was read in its entirety a second time to validate the decision to include the paper in the final data set.

The following inclusion criteria were used to determine the eligibility of each paper:

1) The paper was written in English.

2) The paper addressed the use of a human actors and wearable technology.

3) The paper was published in a peer reviewed scientific journal.

4) The paper was published between the years 1960 and 2019.

5) The paper was not excluded during the quality screen.

6) The paper was available via the University of Eastern Finland Library at no charge.

It should be noted that inclusion criteria \#6 was selected for convenience and practical purposes, however, all databases selected were available within the UEF library and no paper was discovered which had a cost associated with it and thus was excluded.

The date range of 1960 to present day was chosen as this was the year that Howard Barrows introduced standardized patients as a form of health care education (Yudkowsky, 2002). In the years following their introduction, extensive research was conducted regarding the psychometrics of standardized patients (Yudkowsky, 2002).

Each paper was read independently through the lens of the quality screen. As outlined by Okoli and Schabram, each paper was screened for four items: what claims are being made, what evidence is provided to support these claims, if the evidence is warranted, and how the is backed (Okoli \& Schabram, 2010).

\section{Results}

Ten databases were identified as the sources to be used to search for appropriate papers to support this research. The previously identified query was used to search each database. Before the query was ran, a basic query was tested for each database to confirm the unique interpretation of Boolean logic by each database. As a result of this test, the syntax of each query was sometimes modified to produce consistent results. The citations from the result set of each query were saved using the feature of each database to allow for the archiving of each result set. Each database was searched based upon Title, Abstract and Author keywords as defined by the individual database. 
The title, abstract and when necessary the full paper was reviewed to determine if the paper met the inclusion criteria. During phase two, each paper was read in its entirety to ensure that all inclusion criteria was met to arrive at the final result set shown in Table 1. Multiple reviews of each paper through the lens of the inclusion criteria produced the results found in column 4 of Table 1 . It should be noted that a number of the papers that did not fit the inclusion criteria are referenced in this paper as they inform the landscape of health care education using High Fidelity simulators and standardized patients. The nine papers identified are marked in the references section with an asterisk.

\section{Health care disciplines represented in past research}

Of the initial 39 papers from phase one, many health care disciplines were represented covering a broad spectrum of health care areas. However, as can be seen from Table 2, the majority of the papers focused on nursing education.

For each review phase the authors identified the health care discipline in which the paper and associated research was focused upon. From the Table 2 it can be seen that Nursing Education was the focus of the largest single percentage of studies identified in phase $1(28 \%)$ with Physician Training being the next largest at $21 \%$. The other disciplines were represented in just one or two papers, positioning physician and nursing training as representing almost half of the phase 1 papers (Table 3). The final nine papers selected for this systematic literature review were as follows:

Table three outlines the final nine papers selected as the outcome of the systematic literature review. It is interesting to note, yet not surprising, that the majority of the papers were published within the last 3 years, an indication of the novelty of this approach. In alignment with table two, one should also note that the majority of papers represent the nursing education field.

Table 1 Result-set size for each database for each search phase

\begin{tabular}{llll}
\hline Database & $\begin{array}{l}\text { Paper count - initial search } \\
\text { phase }\end{array}$ & $\begin{array}{l}\text { Paper count - phase 1 } \\
\text { inclusion criteria }\end{array}$ & $\begin{array}{l}\text { Paper count - phase 2 } \\
\text { inclusion criteria }\end{array}$ \\
\hline IEEE & 9 & 2 & 0 \\
ACM & 14 & 3 & 0 \\
Science Direct & 53 & 9 & 2 \\
Springer Link & 740 & 4 & 1 \\
Scopus & 361 & 15 & 4 \\
PubMed & 245 & 2 & 0 \\
Web of & 166 & 1 & 0 \\
Science & & & 2 \\
CINAHL & 424 & 3 & 0 \\
Cochrane & 78 & 0 & 0 \\
Library & & 0 & $\mathbf{9}$ \\
EMBASE & 286 & $\mathbf{3 9}$ & \\
Total & $\mathbf{2 3 7 6}$ & &
\end{tabular}


Table 2 Health care disciplines represented

\begin{tabular}{lll}
\hline Health care discipline & $\begin{array}{l}\text { Health care disciplines represented }- \\
\text { phase 1 }\end{array}$ & $\begin{array}{l}\text { Health care disciplines represented - } \\
\text { phase 2 }\end{array}$ \\
\hline Child rehabilitation & 1 & 0 \\
Physiotherapy & 1 & 0 \\
Nursing education & 11 & 3 \\
Emergency medicine & 2 & 0 \\
Disaster medicine & 2 & 0 \\
Neo-natal care & 1 & 0 \\
Physician training & 8 & 1 \\
Trauma team training & 1 & 0 \\
Ultrasonography & 1 & 1 \\
Psychiatry & 1 & 0 \\
Paramedic training & 1 & 1 \\
Acute care training & 1 & 0 \\
Midwifery & 2 & 1 \\
Haemodialysis & 1 & 1 \\
Military physician & 1 & 0 \\
training & 1 & $\mathbf{9}$ \\
Other & $\mathbf{3 9}$ & 1 \\
Pharmacy training & 2 & 0 \\
Not applicable & 1 & 0 \\
Total & &
\end{tabular}

\section{Forms of hybrid simulation identified in the literature review}

Hybrid simulation is a growing form of simulation in health care education. Today, the primary form of simulation is the use of full body mannequins or high fidelity simulators. These types of simulators present to the student a technology based representative of a human body/person that would allow the student to conduct invasive procedures in which the mannequin would 'respond'. However, this approach lacks in the realism which may be required to encourage student to patient interaction. Hybrid simulations generally fall into the category of a worn device such as a sleeve or chest plate that allows for invasive procedures, a silicon overlay to present to the student a particular look or feel or wearable sensors that are used in conjunction with other technology to provide feedback to the student. A novel yet inexpensive approach to hybrid simulation was fashioned by researchers at the University of the Sunshine Coast, Queensland, Australia. The Wearable Simulated Maternity Model, for example, provides a costeffective and realistic alternative that, when worn by simulated patients, enhances fidelity and student ability to practice performing physical examinations ("Andersen et al., 2019).

Researchers at the University of Delaware developed a tracheostomy overlay system (TOS) that is worn by the patient to allow students to conduct tracheostomy suctioning and wound care ("Cowperthwait et al., 2015). Cowperthwait believes that tracheostomy suctioning is an important skill nurses as well as family members need to know ("Holtschneider, 2017). The current practice of suctioning a plastic manikin does not translate to real life, whereas a wearable simulator enables valuable feedback, feedback which a manikin cannot provide ("Holtschneider, 2017). Cowperthwait believes that 
Table 3 Final paper selection

\begin{tabular}{|c|c|c|c|}
\hline Paper title & $\begin{array}{l}\text { Year } \\
\text { published }\end{array}$ & Discipline & Author \\
\hline $\begin{array}{l}\text { Wearable simulated maternity model: Making } \\
\text { simulation encounters real in midwifery }\end{array}$ & 2019 & Midwifery & $\begin{array}{l}\text { Andersen, P., Downer, T., O'Brien, S., \& } \\
\text { Cox, K }\end{array}$ \\
\hline $\begin{array}{l}\text { Tracheostomy Overlay System: An Effective Learning } \\
\text { Device Using Standardized Patients }\end{array}$ & 2015 & $\begin{array}{l}\text { Nursing } \\
\text { Education }\end{array}$ & $\begin{array}{l}\text { Cowperthwait, A. L., Campagnola, N., } \\
\text { Doll, E. J., Downs, R. G., Hott, N. E., Kelly, } \\
\text { S. C., .. Buckley, J. M. }\end{array}$ \\
\hline $\begin{array}{l}\text { An easy-to-build, low-budget point-of-care ultra- } \\
\text { sound simulator: from Linux to a web-based solution }\end{array}$ & 2017 & Ultrasonography & $\begin{array}{l}\text { Damjanovic, D., Goebel, U., Fischer, B., } \\
\text { Huth, M., Breger, H., Buerkle, H., \& } \\
\text { Schmutz, A. }\end{array}$ \\
\hline $\begin{array}{l}\text { Avstick: An Intravenous Catheter Insertion Simulator } \\
\text { for Use with Standardized Patients }\end{array}$ & 2018 & $\begin{array}{l}\text { Nursing } \\
\text { Education }\end{array}$ & $\begin{array}{l}\text { Devenny, A., Lord, D., Matthews, J., } \\
\text { Tuhacek, J., Vitlip, J., Zhang, M., ... } \\
\text { Cowperthwait, A }\end{array}$ \\
\hline $\begin{array}{l}\text { Advancing renal education: hybrid simulation, using } \\
\text { simulated patients to enhance realism in } \\
\text { haemodialysis education }\end{array}$ & 2015 & Haemodialysis & $\begin{array}{l}\text { Dunbar-Reid, K., Sinclair, P. M., \& Hudson, } \\
\text { D. }\end{array}$ \\
\hline $\begin{array}{l}\text { Expanding the Fidelity of Standardized Patients in } \\
\text { Simulation by Incorporating Wearable Technology }\end{array}$ & 2017 & $\begin{array}{l}\text { Nursing } \\
\text { Education }\end{array}$ & Holtschneider, M. E. \\
\hline $\begin{array}{l}\text { Quantitative Approach Based on Wearable Inertial } \\
\text { Sensors to Assess and Identify Motion and Errors in } \\
\text { Techniques Used during Training of Transfers of } \\
\text { Simulated c-Spine-Injured Patients. }\end{array}$ & 2018 & $\begin{array}{l}\text { Paramedic } \\
\text { Training }\end{array}$ & Lebel, K., Chenel, V., Boulay, J., \& Boissy, P. \\
\hline $\begin{array}{l}\text { Hybrid Simulation in Teaching Clinical Breast } \\
\text { Examination to Medical Students }\end{array}$ & 2019 & $\begin{array}{l}\text { Nursing } \\
\text { Education }\end{array}$ & $\begin{array}{l}\text { Nassif, J., Sleiman, A.-K., Nassar, A. H., \& } \\
\text { Naamani, S. }\end{array}$ \\
\hline $\begin{array}{l}\text { High Fidelity Patient Silicone Simulation: A } \\
\text { qualitative evaluation of nursing students' } \\
\text { experiences. }\end{array}$ & 2012 & $\begin{array}{l}\text { Nursing } \\
\text { Education }\end{array}$ & $\begin{array}{l}\text { Reid-Searl, K., Happell, B., Vieth, L., \& } \\
\text { Eaton, A. }\end{array}$ \\
\hline
\end{tabular}

this feedback is critical in increasing learner competency while at the same time preparing both staff and family members for patient reactions when tracheostomy suctioning is being performed ("Holtschneider, 2017).

The TOS was developed by an interdisciplinary team of faculty and students from three departments (engineering, nursing, and theatre) to address the limitations of using a standardized patient in simulation. The TOS sits over the actor's torso, aesthetically representing a chest and throat with an inserted tracheostomy tube. This overlay system allows nursing students to perform tracheostomy care, assessment and suctioning on a live patient. The actor is able to respond accordingly to abnormal suctioning or too much faceplate pressure/manipulation based upon cues provided by sensors within the TOS that can be felt by the actor ("Cowperthwait et al., 2015).

The TOS is worn by a human actor with the intent to improve the procedural techniques of students that are practicing assessment and care of a patient with a tracheostomy ("Cowperthwait et al., 2015). The current use of standardized patients in simulation has been proven to be an effective way to increase scenario realism; however, there are many limitations to the type of injury or illness that can be assigned to standardized patient cases ("Cowperthwait et al., 2015). The use of medical lines on a standardized patient for example is not practical; however some high-fidelity mannequins have the capability to receive a medical line in various parts of their anatomy. However, these mannequins lack the ability to interact with the caregiver and elicit the necessary emotions and body language that a real patient would naturally present to the care-giver.

Another approach found in the literature of hybrid simulation is to outfit the standardized patient with a wearable sleeve which would allow the student to perform invasive procedures such as inserting an IV into the arm that could be leveraged for various 
healthcare training scenarios. This approach was used by a group of researchers at the University of Delaware and similarly by a group of researchers from Australia. A second group of researchers, also from the University of Delaware, used a wearable sleeve to develop Avstick, an Intravenous Catheter insertion simulator for use with standardized patients ("Devenny et al., 2018). This device allows the nurse trainee to perform an intravenous catheter insertion on a live patient without causing harm or stress to the patient. Whereas Dunbar-Reid et al. used the wearable sleeve to enhance realism in haemodialysis training ("Dunbar-Reid et al., 2015). This wearable sleeve simulator allowed a standardized patient to be 'dialysed'.

Silicon is another common material used by researchers to re-produce parts of the body to either present to the learner visual cues or tactile surfaces to assess. A group of researchers from CO University Australia developed the persona of a simulated patient complete with a personal and medical history. This simulated patient was then "brought to life' by the professor who donned life-like silicone props which represented face, hands and torso. The professor, in character, interacted with the students and answered questions as the patient, and posed new questions for the students to consider and to guide the discussion ("Reid-Searl, Happell, Vieth, \& Eaton, 2012). Similarly, researchers from Universities in Lebanon and the United States co-developed a hybrid teaching model in which clinical breast exams were conducted on a standardized patient wearing a silicone breast simulator jacket ("Nassif, Sleiman, Nassar, \& Naamani, 2019). This silicon prop presented to the learner a silicon based breast with integrated lesions, which allowed the learner to conduct a clinical breast exam that realistically represented a live patient.

Remote sensors are another common element of hybrid simulation. These sensors are strategically placed on various parts of the body of the standardized patient. The sensors are then integrated with external technology to provide the learner with some form of electronic feedback that becomes part of the learning scenario. Researchers from the Department of Anesthesiology and Critical Care, Medical Center-University of Freiburg, Faculty of Medicine, at the University of Freiburg, developed a more affordable and accessible hybrid training approach to deliver hands on training in point of care ultrasound systems, which are often used for the initial clinical assessment of critically ill patients. Researchers developed an HTML browser-based ultrasound simulation application based upon the original Linux based version developed by Kulyk and Olsynski in 2011. This application reads inputs from sensors that are attached to standardized patients ("Damjanovic et al., 2017). Similarly, Canadian researchers explored the use of wearable inertial sensors to assess and identify motion and errors in techniques used during transfers of simulated c-spine injured patients. These wearable sensors provided the trainees with objective feedback along with a three dimensional model of the performed move, providing specific areas of improvement for future transfer attempts.

\section{Lessons learned from hybrid simulations identified in the literature review}

A common theme identified in the literature as it relates to hybrid simulation is the improvement in trainee-patient interaction as a result of having a human actor as part of the simulation. This compared to simulations based upon mannequins alone, where 
students often raised concerns about the lack of realism of the simulation due to the lack of interaction with a 'real person'. This lack of interaction is significantly overcome by the use of standardized patients as they can speak and readily display nonverbal behavior in reaction to what learners do and say ("Holtschneider, 2017).

Cowperthwait et al. for example found that the use of the tracheostomy overlay system demonstrated significantly more positive clinical interactions than the mannequin based scenario ("Cowperthwait et al., 2015). In addition to an increased amount of positive patient interactions, students who trained with the tracheostomy overlay system self-corrected their behavior considerably more than those who trained with the mannequin ("Cowperthwait et al., 2015). A similar result was seen by Dunbar-Reid et al. who used hybrid simulation in haemodialysis education. This simulation enabled participants to practice clinical skills relative to renal patient care while simultaneously developing communication skills while interacting with the human actor ("Dunbar-Reid et al., 2015). Researchers found that the hybrid simulation approach delivered enhanced realism and therefore provided a more authentic learning context without putting real patients at risk ("Dunbar-Reid et al., 2015). The renal-specific hybrid-based simulation approach provided students with an authentic, patient centered environment that allowed instructors to assess student's technical and interpersonal competencies.

Similarly, Devenny et al. found that by using Avstick, an Intravenous Catheter Insertion Simulator, trainee-patient communication, procedure explanation, patient reassurance, question asking, and general patient interaction, showed a significant increase as compared to the same group being trained using a mannequin ("Devenny et al., 2018). Researchers concluded from these results that the wearable IV trainer, Avstick, is as effective as a mannequin for improving student self-efficacy and is superior to training with a mannequin as it relates to improving student interaction with the patient during clinical encounters. Indeed, anecdotal evidence clearly showed that students were much more willing to respond to and engage in conversation with a human actor wearing the Avstick than with a 'static representation of a human patient' ("Devenny et al., 2018).

Other hybrid simulation studies showed similar positive results. However, not all results were tied to communications. Reid-Searl et al. found that the use of silicon props worn by a standardized patient, in this case the professor, took students out of their comfort zone which in turn reduced their fear and increased their self-confidence, which the students felt better prepared them for future clinical placements ("Reid-Searl et al., 2012). During the debriefing, students described how this simulation experience helped them to build confidence in their ability to work with real human beings in the workplace thus reducing some of their fears of this inevitable reality ("Reid-Searl et al., 2012). Indeed, many of the participants described the simulation as taking them 'out of their comfort zone' and forcing them to actively engage with the patient ("Reid-Searl et al., 2012). One of the obvious advantages of this approach was the reduction of risk in using a human actor vs a real patient, this significantly reduced the fear of harming the patient through inappropriate actions or behaviour. The researchers concluded that these findings highlight important considerations for nursing education around active learning, reducing anxiety and encouraging students to regard patients as real human beings rather than focusing primarily on symptoms and techniques ("Reid-Searl et al., 2012). Similarly, Nassif et al. found that hybrid simulation using silicon breast jackets produced significantly higher lesion reporting, identification of malignant features, and 
accurate location identification as compared to the traditional teaching methods ("Nassif et al., 2019). Indeed, students in the hybrid simulation group indicated, through satisfaction surveys, that they were more likely to recommend hybrid simulation for teaching clinical breast examination, that hybrid simulation helped develop confidence in the clinical setting and that the hybrid simulation helped to integrate the theory of a clinical breast examination with the practice ("Nassif et al., 2019).

In regards to wearable sensors, Lebel et al. found that the use of motion sensors affixed to standardized patients allowed researchers to provide more specific, quality feedback to learners enabling them to more easily correct emergency rolling techniques performed on c-spine injured patients. Researchers found that the use of wearable inertial sensors provided instructors with objective data to provide personalized feedback during training and could be further employed to provide a complete training solution by directly embedding the inertial sensors into mannequins ("Lebel, Chenel, Boulay, \& Boissy, 2018).

Damjanovic et al. also showed that the use of embedded sensors can be useful in emergency medical situations. This hybrid simulation approach demonstrated that a robust ultrasound simulator can be fabricated for a fraction of the cost of commercially available solutions, making this a novel approach for ultrasound education in developing countries. Additionally, this technology may be applied in situations where a casualty surge is experienced, as point of care ultrasound has been shown to aid in the management of mass casualties, such as those experienced during the Boston bombings. Due to the solutions low cost and lack of required hardware, as the solution is primarily a software solution, researchers felt that this design could be easily employed in blended learning environments facilitating the savings of time and resources.

Through the use of the Wearable Simulated Maternity Model, Andersen et al. found that students enjoyed the authentic immersive approach to midwifery simulation using real people to practice clinical and communication skills, rather than inanimate objects such as manikins or part task training models ("Andersen et al., 2019). Indeed, the Wearable Simulated Maternity Model has shown that a simple to implement simulation experience can be designed that provides a high-fidelity simulation at a very low cost ("Andersen et al., 2019). This model was fabricated using readily available yet inexpensive materials ("Andersen et al., 2019). Anderson et al. found that despite the "low budget' production, the implementation of this model in a student simulation scenario showed a notable impact on student learning and engagement ("Andersen et al., 2019).

A significant, yet often overlooked advantage of hybrid simulation is the ability to incorporate diversity into our simulation scenarios ("Holtschneider, 2017). Indeed, a problem identified by Cowperthwait is that many of the manikins currently on the market have Caucasian features but have black skin, which is not realistic ("Holtschneider, 2017). Alternatively, hybrid simulation models allow the standardized patient to be whoever they are, allowing the educator to use a diverse population, allowing them to speak for themselves ("Holtschneider, 2017).

\section{Future research direction}

The general theme of this research was the question of how health care education can be enhanced through the use of wearable technology and human actors. To completely 
answer this question more longitudinal research is required to understand how hybrid simulation techniques enable health care workers to perform their duties more effectively in the field as compared to training based upon high fidelity simulators or standardized patients only.

Additionally, more work is required to better understand, and indeed maximize the way in which standardized patients can provide appropriate verbal feedback to learners to help them improve communication skills and how this focus on communication can promote a patient-centered care model ("Holtschneider, 2017). However, this 'appropriate' verbal feedback may not come naturally to the standardized patient. Indeed, Cowperthwait et al. found that during the tracheostomy care scenario standardized patients did not know how to appropriately react to suctioning that was too deep unless they were properly trained ("Holtschneider, 2017). This training came in the form of interviews with former tracheostomy patients, allowing the standardized patients to hear firsthand the patients' thoughts, feelings, and emotions ("Holtschneider, 2017). More work is required to explore the impact of various approaches to standardized patient training, and how this training is reflected in the fidelity of the simulation and thus the long term efficacy of the learner.

In her work with the University of Delaware, Cowperthwait discovered that it is not only the learner that benefits from the use of standardized patients, but the standardized patients themselves ("Holtschneider, 2017). Through the simulation scenarios, Cowperthwait found that standardized patients have become better patient advocates when they and their family members receive health care ("Holtschneider, 2017). This insight opens opportunity for further research to better understand the depths and types of reciprocal benefits of using standardized patients during simulation scenarios and its impact on the broader patient care environment ("Holtschneider, 2017).

Finally, the use of wearable devices opens up many avenues for learners to practice critical care interventions. More work is required to explore what other intervention based procedures can be simulated using a hybrid simulation model ("Holtschneider, 2017).

\section{Conclusion}

Health-care education using simulation technology is a much diversified field covering all aspects of the health care industry. Generally speaking, health care education simulation is implemented using four general approaches: stand-alone high fidelity simulators, stand-alone standardized patients, virtual patients and hybrid simulation, where technology is integrated with human actors to present a hybrid training scenario to the student. Based upon the literature, hybrid simulation appears to fall into three general categories: technology based overlays which allow for intrusive procedures on a human actor, wearable sensors which provide feedback to both the trainee and the human actor, and silicon overlays which present to the trainee a visual and/or tactile appendage in which the trainee can assess. In most cases, hybrid simulation performs equally as well as high fidelity simulators in allowing the trainee to practice procedural and declarative knowledge. However, in all cases the hybrid simulation presents the student with a superior learning environment to practice patient to care-giver interaction. The presence of the human actor elicits more procedure explanation, patient reassurance, 
asking of questions by the caregiver, and in general more patient interaction. All of which are almost non-existent when high fidelity simulators are used.

Although not directly evidenced in the literature, one of the main disadvantages of hybrid simulation is the need for trained actors. In certain scenarios these actors may have a cost associated with them which will impact the cost effectiveness of a hybrid approach. However, as illustrated by Cowperthwait et al. there may be willing actors found at no cost within the learning institution if the institution has a theatre program (*Cowperthwait et al., 2015). Additionally and again not directly evidenced in the literature, the use of human actors puts one at the mercy of the availability and willingness of these actors to fulfill the role required within the scenario. This is where the 24/7 availability of a high fidelity simulator outshines the human actor in availability, however, a high fidelity simulator usually requires the presence of at least one simulator technician to ensure the smooth operation of the device.

Technology based hybrid simulation has been shown to be flexible in its ability to simulate a variety of invasive and non-invasive health care scenarios. This literature review illustrates that there is significant opportunity for the expansion of the role of hybrid simulation in health care education, a role which should improve learner competence and confidence.

Acknowledgements

Not applicable.

Authors' contributions

The author(s) read and approved the final manuscript.

Funding

Not applicable.

Availability of data and materials

The datasets used and/or analysed during the current study are available from the corresponding author on reasonable request.

Competing interests

The authors declare that they have no competing interests.

Author details

${ }^{1}$ University of Eastern Finland, Yliopistokatu 2, Joensuu FI-80100, Finland. ${ }^{2}$ College of Information, University of North Texas, UNT Discovery Park, 3940 North Elm, Suite C232, Denton, TX 76203-5017, USA.

Received: 26 March 2020 Accepted: 3 June 2020

Published online: 12 June 2020

References

${ }^{*}$ Papers of past research directly related to hybrid simulation

*Andersen, P., Downer, T., O'Brien, S., \& Cox, K. (2019). Wearable simulated maternity model: making simulation encounters real in midwifery. Clinical Simulation in Nursing, 33(C), 1-6. https://doi.org/10.1016/j.ecns.2019.04.007.

Amerjee, A., Akhtar, M., Ahmed, I., \& Irfan, S. (2018). Hybrid simulation training: an effective teaching and learning modality for intrauterine contraceptive device insertion. Education and Health, 31, 119-124. https://doi.org/10.4103/efh.EfH_357_17.

Bloice, M. D., et al. (2013). On the usage of health records for the design of virtual patients: a systematic review. BMC Medical Informatics and Decision Making, 13(1), 103. https://doi.org/10.1186/1472-6947-13-103.

*Cowperthwait, A. L., Campagnola, N., Doll, E. J., Downs, R. G., Hott, N. E., Kelly, S. C., et al. (2015). Tracheostomy overlay system: an effective learning device using standardized patients. Clinical Simulation in Nursing, 11(5), 253-258. Retrieved from. https://doi.org/10.1016/j.ecns.2015.03.001.

*Damjanovic, D., Goebel, U., Fischer, B., Huth, M., Breger, H., Buerkle, H., \& Schmutz, A. (2017). An easy-to-build, low-budget point-of-care ultrasound simulator: from Linux to a web-based solution. Critical Ultrasound Journal, 9(4), 1-6. https://doi. org/10.1186/s13089-017-0061-4.

*Devenny, A., Lord, D., Matthews, J., Tuhacek, J., Vitlip, J., Zhang, M., et al. (2018). Avstick: an intravenous catheter insertion simulator for use with standardized patients. Nursing Education Perspectives, 39(2), 102-104. https://doi.org/10.1097/01. NEP.0000000000000225.

*Dunbar-Reid, K., Sinclair, P. M., \& Hudson, D. (2015). Advancing renal education: Hybrid simulation, using simulated patients to enhance realism in haemodialysis education. Journal of Renal Care, 41(2), 134-139. 
EBSE. (2007). Guidelines for performing systematic literature reviews in software engineering. guidelines for performing systematic literature reviews in software engineering (Vol. 01, pp. 1-57). Keele.

Goolsby, C. A., Goodwin, T. L., \& Vest, R. M. (2014). Hybrid simulation improves medical student procedural confidence during EM clerkship. Military Medicine, 179, 1223-1227. https://doi.org/10.7205/MILMED-D-14-00072.

*Holtschneider, M. E. (2017). Expanding the Fidelity of standardized patients in simulation by incorporating wearable technology. Journal for Nurses in Professional Development, 33(6), 320-321. https://doi.org/10.1097/nnd. 0000000000000391.

Ignacio, J., Dolmans, D., Scherpbier, A., Rethans, J.-J., Chan, S., \& Liaw, S. Y. (2015). Comparison of standardized patients with high-fidelity simulators for managing stress and improving performance in clinical deterioration: A mixed methods study. Nurse Education Today, 35, 1161-1168. Retrieved from. https://doi.org/10.1016/j.nedt.2015.05.009.

Kennedy, J. L., Jones, S. M., Porter, N., White, M. L., Gephardt, G., Hill, T., \& Thompson, T. M. (2013). High-Fidelity hybrid simulation of allergic emergencies demonstrates improved preparedness for office emergencies in pediatric allergy clinics. The Journal of Allergy and Clinical Immunology. In Practice, 1, 608-617. https://doi.org/10.1016/j.jaip.2013.07.006.

*Lebel, K., Chenel, V., Boulay, J., \& Boissy, P. (2018). Quantitative approach based on wearable inertial sensors to assess and identify motion and errors in techniques used during training of transfers of simulated c-spine-injured patients. Journal of Healthcare Engineering, 2018, 1-9. https://doi.org/10.1155/2018/5190693.

Latif, R., Abbas, H., \& Assar, S. (2014). Distributed denial of service (DDoS) attack in cloud- assisted wireless body area networks: A systematic literature review. Journal of Medical Systems, 38, 1-10. https://doi.org/10.1007/s10916-014-0128-8.

Lawrence, D. W. (2008). What is lost when searching only one literature database for articles relevant to injury prevention and safety promotion? Injury Prevention, 14, 401-404. https://doi.org/10.1136/ip.2008.019430.

Lous, M. L., et al. (2020). Hybrid simulation for obstetrics training: a systematic review. European Journal of Obstetrics \& Gynecology and Reproductive Biology, 246, 23-28. https://doi.org/10.1016/j.ejogrb.2019.12.024.

Luctkar-Flude, M., Wilson-Keates, B., \& Larocque, M. (2012). Evaluating high-fidelity human simulators and standardized patients in an undergraduate nursing health assessment course. Nurse Education Today, 32, 448-452. https://doi.org/10. 1016/j.nedt.2011.04.011.

*Nassif, J., Sleiman, A.-K., Nassar, A. H., \& Naamani, S. (2019). Hybrid simulation in teaching clinical breast examination to medical students. Journal for Cancer Education, 34, 194-200. https://doi.org/10.1007/s13187-017-1287-3.

O'Mara-Eves, A., Thomas, J., McNaught, J., Miwa, M., \& Ananiadou, S. (2015). Using text mining for study identification in systematic reviews: A systematic review of current approaches. Systematic Reviews, 4(5), 1-22. https://doi.org/10.1186/ 2046-4053-4-5.

Okoli, C., \& Schabram, K. (2010). A guide to conducting a systematic literature review of information systems research. Sprouts: Working Papers on Information Systems, 10(26) http://sprouts.aisnet.org/10-26.

Qi, X., Yang, M., Ren, W., Jia, J., Wang, J., Han, G., \& Fan, D. (2013). Find duplicates among the PubMed, EMBASE, and Cochrane library databases in systematic review. PLoS One, 8(8), 1-12. https://doi.org/10.1371/journal.pone.0071838.

*Reid-Searl, K., Happell, B., Vieth, L., \& Eaton, A. (2012). High fidelity patient silicone simulation: a qualitative evaluation of nursing students' experiences. Collegian, 19, 77-83. https://doi.org/10.1016/j.colegn.2011.09.003.

Rosen, K. R. (2008). The history of medical simulation. Journal of Critical Care, 23, 157-166. https://doi.org/10.1016/j.jcrc.2007. 12.004.

Sanko, J., Shekhter, I., Rosen, L., Arheart, K., \& Birnbach, D. (2012). Man versus machine: the preferred modality. The Clinical Teacher, 9, 387-391. https://doi.org/10.1111/j.1743-498X.2012.00593.x.

Schubart, J. R., Erdahl, L., Smith, S. J., Purichia, H., Kauffman, G. L., \& Kass, R. B. (2012). Use of breast simulators compared with standardized patients in teaching the clinical breast examination to medical students. Journal of Surgical Education, 69(3), 416-422. https://doi.org/10.1016/j.jsurg.2011.10.005.

Smithburger, P. L., Kane-Gill, S. L., Ruby, C. M., \& Seybert, A. L. (2012). Comparing effectiveness of 3 learning strategies simulation-based learning, problem-based learning, and standardized patients. Simulation in Healthcare, 7(3), 141-146. https://doi.org/10.1097/SIH.0b013e31823ee24d.

Tuzer, H., Dinc, L., \& Elcin, M. (2016). The effects of using high-fidelity simulators and standardized patients on the thorax, lung, and cardiac examination skills of undergraduate nursing students. Nurse Education Today, 45, 120-125. https://doi. org/10.1016/j.nedt.2016.07.002.

Verma, A., Bhatt, H., Booten, P., \& Kneebone, R. (2011). The Ventriloscope as an innovative tool for assessing clinical examination skills: appraisal of a novel method of simulating auscultatory findings. Medical Teacher, 33, 388-396. https:// doi.org/10.3109/0142159X.2011.579200.

Wallace, D., Gillett, B., Wright, B., Stetz, J., \& Arquilla, B. (2010). Randomized controlled trial of high fidelity patient simulators compared to actor patients in a pandemic influenza drill scenario. Resuscitation, 81, 872-876. https://doi.org/10.1016/j. resuscitation.2010.02.026.

Wisborg, T., Brattebo, G., Brinchmann-Hansen, A., \& Hansen, K. S. (2009). Mannequin or standardized patient: participants' assessment of two training modalities in trauma team simulation. Scandinavian Journal of Trauma, Resuscitation and Emergency Medicine, 17(59), 1-4. https://doi.org/10.1186/1757-7241-17-59.

Yudkowsky, R. (2002). Should we use standardized patients instead of real patients for high-stakes exams in psychiatry? Academic Psychiatry, 26(3), 187-192.

\section{Publisher's Note}

Springer Nature remains neutral with regard to jurisdictional claims in published maps and institutional affiliations. 